\title{
Cell Dimensioning and Network Throughput in Cellular Multi-Hop Relay Networks
}

\author{
K.R. Jacobson, W.A. Krzymień \\ TRLabs/Electrical and Computer Engineering, University of Alberta \\ Edmonton, Alberta, Canada \\ krj@ualberta.ca, wak@ece.ualberta.ca
}

\begin{abstract}
In this paper we look at the network throughput and efficiency of a cellular system employing multi-hop relaying. It is shown that multi-hop relaying can provide great improvement in the area averaged spectral efficiency (measured in bits/second/Hz per unit area of coverage) of a system. However, the number of relays introduced and the dimensions of coverage areas of relays must be carefully chosen for the specific propagation environment and system parameters, otherwise poorer efficiency may result. Some example designs for the $5 \mathrm{GHz}$ unlicensed band are given to illustrate some design considerations. Realistic simulated throughput, considering physical and medium access control layer overhead, of a HiperMAN or 802.16 system together with noise and interference calculations are used to determine potential real-world performance of such systems. Calculations for one to four relay hops are presented and designs' area averaged spectral efficiencies are compared.
\end{abstract}

Keywords: cellular networks, multi-hop relaying, WiMAX, HiperMAN, throughput, dimensioning.

\section{INTRODUCTION}

Multi-hop relaying [1], [2], [3] is a key technique for increasing data transmission rates and maximizing coverage in future generation wireless data systems. With relaying, data destined for a mobile terminal will be relayed to a wireless relay station nearest the terminal rather than being transmitted directly from a more distant base station. Although relaying requires the use of additional radio resources (frequency channels or time slots) it can significantly reduce the path loss by shortening the propagation path and routing around obstacles. Reduced path loss translates to increased spectral efficiency. In addition, spatial reuse (SR) [4], [5] is enabled by using multi-hop relaying. SR allows simultaneous transmissions in the same channel by numerous stations within the macrocell with minimal interference. These effects combine to increase the spectral efficiency and coverage of a wireless relay network. In order to economically achieve the best coverage and network throughput, relay network design must be done carefully, considering distribution of user terminals and the radio propagation environment. Cellular providers have a significant investment in their existing networks, and reuse of existing base stations (BSs) is desirable when converting to new technologies.

Multi-hop relaying is an option in 802.16-2004 (mesh mode) [6] and is studied in 802.16e work (mobile multi-hop relaying mode - MMR) [7], [8]. Similarly it is a key part of High Performance Radio Metropolitan Area Networks (HiperMAN) [9], [10] and Wireless Broadband (WiBRO) [11] standards. In this paper, we look at the design issues when converting an existing cellular system with existing base stations to a multihop relaying system such as 802.16 , HiperMAN or WiBRO. Coverage radius of the base station, placement of relays and their coverage radii, must be determined. In this paper, a simple calculation procedure is used to determine the change in coverage and network throughput as relay hops are added to a macrocell system.

There has been work published on such topics in the $2.4 \mathrm{GHz}$ unlicensed band and the $3.5 \mathrm{GHz}$ licensed band. However, the $2.4 \mathrm{GHz}$ band is very crowded, and many applications require the more cost effective unlicensed spectrum. Consequently, we have chosen to use unlicensed bands in the $5 \mathrm{GHz}$ region for this work.

In Section II, we present the system model, calculated results are discussed in Section III, and Section IV concludes the paper.

\section{SySTEM Model}

The design of a multi-hop relaying system begins with existing macrocells of radius, $r$, each with a base station (BS) at its centre. Both hexagonal and Manhattan deployment environments are considered in this study. The macrocell is then partitioned into numerous microcells, each of which is covered by a relay station (RS). Fig. 1 shows typical hexagonal and Manhattan geometries for a maximum hop number, $n_{\text {hops }}$, of four. Following 802.16-2004 nomenclature, the BS is a basestation which is connected to the wired network, RS is a relay station which provides wireless relaying between the BS and the SS and other RSs, and SS is a wireless subscriber station. Links between BS, RSs, and SSs form a tree topology.

Link budgets for the forward link of each hop of a multihop communication link have been calculated using the parameters summarized in Table I (parameters based on [6], [12], [13]). Noise and interference from other microcells have been included to calculate signal to interference and noise ratio (SINR) on each link (BS-RS, RS-RS, and RS-SS) for various one dimensional, hexagonal and Manhattan layouts. Interference from all microcells in macrocells outside the studied macrocell has been included, since we assume no coordination of transmissions occurs beyond macrocell boundaries. Interference from other microcells within the studied macrocell is included when spatial reuse is employed. Adaptive 


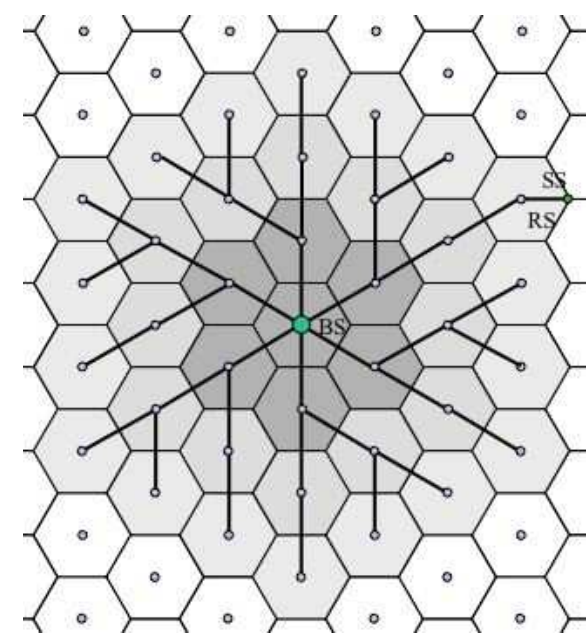

(a) Hex layout - 4 hops.

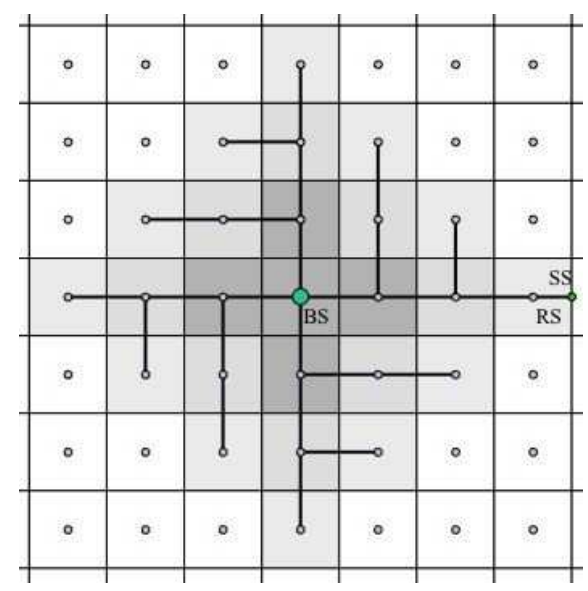

(b) Manhattan layout - 4 hops.

Fig. 1. Cellular relay network topologies for hex and Manhattan layouts.

modulation and coding (AMC) has been applied to determine the throughput achievable for the SINR calculated on each individual link. These link throughputs, together with spatial reuse schedules, are used to calculate the macrocell's aggregate throughput, $R_{\text {net }}$ (bits/second/Hz), and area averaged spectral efficiency (AASE in bits/second/Hz/area) [14], [15]. While most researchers have used $R_{n e t}$ calculations to assess multihop performance, we focus on AASE, which gives a more meaningful measure of the system-wide use of spectrum. In addition, we use realistic achievable throughput with AMC considering overhead.

\section{A. Path Loss Models}

Following recommendations in IEEE 802.16-2004 [16] and the Third Generation Partnership Project (3GPP) [12] the radio frequency (RF) path loss models used in this work are: COST231-Hata model for macrocells (when path length is greater than $1 \mathrm{~km}$ ), and COST231-Walfish-Ikegami for microcells and macrocells when path lengths are less than 1 $\mathrm{km}$. These models are suitable for frequencies up to $2 \mathrm{GHz}$. Many applications will use $5 \mathrm{GHz}$ unlicensed spectrum, and so we have also used extensions to these models given by [13]. With the model parameters summarized in Table I, the resulting line-of-sight (LOS) and non-line-of-sight (NLOS) path loss models used in this work are given in Table III. Shadowing and small scale fading are not explicitly included in these calculations since their effects are averaged out.

Since a key benefit of a multi-hop relay network is its ability to relay around obstacles, network modeling must capture whether the path is LOS or NLOS. We have used a "dualslope" model, in which a NLOS or LOS pathloss model is chosen based on the path length. If the path length is less than a distance breakpoint, then the LOS model is chosen. Otherwise, the NLOS model is chosen. This breakpoint depends on frequency, geometry (exact positions of the stations), and the propagation environment. For the purposes of this work, we can approximate it by looking at a typical urban block, as
TABLE I

MODEL PARAMETERS.

\begin{tabular}{l|l}
\hline \hline \multicolumn{2}{c}{ System Parameters } \\
\hline Carrier frequency & $5.8 \mathrm{GHz}$ \\
Channel bandwidth (W) & $10 \mathrm{MHz}$ \\
Receiver noise figure (F) & $6 \mathrm{~dB}$ \\
Receiver noise floor (kTWF) & $-98 \mathrm{dBm}$ \\
Maximum transmit power & $30 \mathrm{dBm}$ \\
Omni antenna gain & $9 \mathrm{dBi}$ \\
Directional antenna gain & $17.5 \mathrm{dBi}$ \\
Directional antenna front-back ratio & $25 \mathrm{~dB}$ \\
Other losses (cable) & $6 \mathrm{~dB}$ \\
Link margin & $6 \mathrm{~dB}$ \\
Duplexing & TDD \\
Multiple access & TDMA \\
PHY mode & 802.16 OFDM \\
Macrocell BS antenna height & $32 \mathrm{~m}$ \\
Microcell BS antenna height & $10 \mathrm{~m}$ \\
Microcell RS antenna height & $10 \mathrm{~m}$ \\
SS antenna height & $1.5 \mathrm{~m}$ \\
Building height & $12 \mathrm{~m}$ \\
Building to building distance & $50 \mathrm{~m}$ \\
Block size & $200 \mathrm{~m}$ \\
\hline \hline \multicolumn{2}{c}{ Dimensioning Parameters } \\
\hline Macrocell radii $(r)$ & $0.5,1.0,2.0$, and 3.0 km \\
Macrocell cluster size $(N)$ & $1,2,3$, and 4 \\
Number of hops $\left(n_{\text {hops }}\right)$ & $1,2,3$, and 4 \\
Spatial reuse (SR) & with and without \\
Multi-hop path & LOS and NLOS \\
Antenna patterns & Five configurations (Table II) \\
\hline \hline
\end{tabular}

TABLE II

ANTENNA CONFIGURATIONS: O=OMNI, D=DIRECTIONAL.

\begin{tabular}{c|l|l|l}
\hline \hline Configuration (A) & BS Antenna & RS Antenna & SS Antenna \\
\hline 1 & O tx, O rx & O tx, O rx & O tx, O rx \\
2 & D tx, D rx & O tx, O rx & O tx, O rx \\
3 & D tx, D rx & O tx, D rx & O tx, O rx \\
4 & D tx, D rx & D tx, D rx & O tx, O rx \\
5 & D tx, D rx & D tx, D rx & D tx, D rx \\
\hline \hline
\end{tabular}


TABLE III

RF PATH LOSS MODELS, $x$ IS PATH LENGTH IN METRES.

\begin{tabular}{l|c|c}
\hline \hline Environment & $2 \mathrm{GHz}$ Loss $(\mathrm{dB})$ & $5.8 \mathrm{GHz}$ Loss $(\mathrm{dB})$ \\
\hline $\begin{array}{l}\text { Urban Macrocell NLOS } \\
\text { BS to SS, interferers } \\
x>1000 \mathrm{~m}\end{array}$ & $34.5+35.0 \log _{10}(x)$ & $42.5+35.0 \log _{10}(x)$ \\
\hline $\begin{array}{l}\text { Urban Microcell NLOS } \\
\begin{array}{l}\text { BS to RS, RS to SS } \\
5000 \mathrm{~m}>x>\text { breakpoint }\end{array}\end{array}$ & $34.5+38.0 \log _{10}(x)$ & $42.5+38.0 \log _{10}(x)$ \\
\hline $\begin{array}{l}\text { Urban Microcell LOS } \\
\text { BS to RS, RS to SS } \\
20 \mathrm{~m}<x<\text { breakpoint }\end{array}$ & $30.2+26.0 \log _{10}(x)$ & $38.2+26.0 \log _{10}(x)$ \\
\hline \hline
\end{tabular}

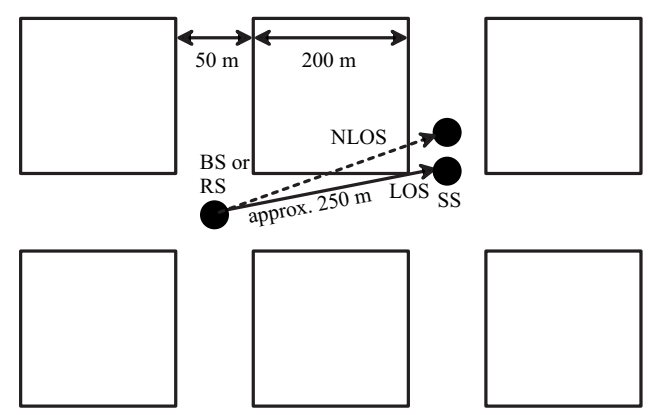

Fig. 2. Distance breakpoint in Manhattan environment.

shown in Fig. 2. An MS has an LOS path to the RS or BS if it is in the street corridor. As it moves behind the corner of a building its path becomes NLOS. At the closest perpendicular street the distance is approximately $250 \mathrm{~m}$. Inter-microcell and inter-macrocell interference is always further away, and thus is always NLOS.

\section{B. Adaptive Modulation and Coding, and Network Through- put}

One of the techniques used to improve the spectral efficiency of broadband wireless networks is adaptive modulation and coding (AMC). The signal to interference and noise ratio (SINR) varies considerably throughout a wireless network, and often between successive transmissions on a particular link. AMC adapts the forward error correction coding (FEC) and modulation scheme used on each burst of information to maximize throughput. Another key technique used in 802.16 and HiperMAN is orthogonal frequency division multiplexing (OFDM).

Table IV shows the modulation and coding for different received SINR values [17]. Column 3 gives AMC thresholds for a coded BER less than $10^{-6}$. Column 4 shows the theoretical spectral efficiency. Obviously this is an upper bound on the spectral efficiency. Each of the PHY and MAC layers add overhead in the frame so that fewer of the transmitted bits carry usable payload. Other implementation inefficiencies must also be considered. Theoretical and simulation work in [17], [18] on HiperLAN, HiperMAN and 802.16 gives more realistic spectral efficiencies for OFDM. Columns 5 and 6 (derived from the results in [18]) are the spectral efficiencies calculated for the PHY and MAC layers considering overhead, and column 7 gives the overall spectral efficiencies obtained via simulation of an 802.16/HiperMAN system. The values in column 7 were used to calculate the network throughput presented here.

When calculating the total network throughput, we must consider the rate achievable on each hop, and the scheduling of transmissions among the network nodes. Relaying incurs inefficiency since additional radio resources (TDMA or FDMA slots) must be used to relay data. This is taken into consideration in throughput calculations. In this paper, network throughput for one macrocell is calculated by

$$
R_{\text {net }}=\frac{n_{\text {delivered }}}{T_{\text {delivery }} \cdot W} \quad b / s / H z
$$

where $n_{\text {delivered }}$ is the number of bits delivered to subscribers, $T_{\text {delivery }}$ is the total time required to deliver the bits (includes the time required for relaying and scheduling), and $W$ is the bandwidth used. An important measure of the coverage of a network is area averaged spectral efficiency (AASE) [14], [15], given by

$$
A A S E=\frac{R_{n e t}}{A \cdot N} \quad\left(b / s / H z / k m^{2}\right)
$$

where $A$ is the area of the macrocell, and $N$ is the macrocell cluster size.

\section{Calculated Results}

Numerous tables of $S I N R, R_{n e t}$ and $A A S E$ values have been calculated for the dimensioning design cases listed in Table I. The results are best presented using a couple of examples comparing various similar designs.

For a macrocell radius of $r=1 \mathrm{~km}$, a set of designs chosen for comparison are shown in Table V. Note that the addition of relaying, with $n_{\text {hops }}=2$, worsens the network throughput and $A A S E$. Since two hop neighbors must coordinate (in 802.16-2004), no spatial reuse is possible. The addition of a second relay $\left(n_{\text {hops }}=3\right)$ increases throughput and $A A S E$ when spatial reuse is employed. The big gains appear if the relays create LOS paths by relaying around obstacles. In this example, the $A A S E$ is improved by $585 \%$ over the single hop case by using 2 relays if the paths are LOS and spatial reuse is employed.

Note that in all of the above cases, the use of directional transmit and receive antennas for all nodes (BS, RS, and SS) achieves the best performance. Often it is more practical for the SS to have an omnidirectional antenna. Table VI shows a number of designs chosen with an omnidirectional SS antenna. For the $n_{\text {hops }}=1$ reference case, the SINR does not meet the minimum required for even the lowest order AMC scheme, and thus the throughput is zero. Adding a relay, even with NLOS paths, increases the SINR to meet the minimum and thus makes communication possible. So in this case, $n_{\text {hops }}=$ 2 is beneficial. If the paths are NLOS, then four relay hops are required to get the best $A A S E$, whereas only three were required in the previous set of design examples. With LOS, three hops suffice as before, but a cluster size of two is required instead of one. 
TABLE IV

AMC IN 802.16 FOR BER THRESHOLD OF $10^{-6}$ (ADAPTED FROM [6], [17] AND [18]).

\begin{tabular}{l|c|c|c|c|c|c}
\hline \hline Modulation & Code Rate & $\begin{array}{c}\text { SINR Thresh. } \\
(\mathrm{dB})\end{array}$ & $\begin{array}{c}\text { Theor. Rate } \\
(\mathrm{b} / \text { channel use })\end{array}$ & $\begin{array}{c}\text { PHY Rate } \\
(\mathrm{b} / \mathrm{s} / \mathrm{Hz})\end{array}$ & $\begin{array}{c}\text { MAC Rate } \\
(\mathrm{b} / \mathrm{s} / \mathrm{Hz})\end{array}$ & $\begin{array}{c}\text { Sim. Rate } \\
(\mathrm{b} / \mathrm{s} / \mathrm{Hz})\end{array}$ \\
\hline BPSK & $1 / 2$ & 6.4 & 0.5 & 0.35 & 0.31 & 0.29 \\
QPSK & $1 / 2$ & 9.4 & 1.0 & 0.69 & 0.61 & 0.59 \\
QPSK & $3 / 4$ & 11.2 & 1.5 & 1.04 & 0.93 & 0.88 \\
16QAM & $1 / 2$ & 16.4 & 2.0 & 1.38 & 1.23 & 1.19 \\
16QAM & $3 / 4$ & 18.2 & 3.0 & 2.07 & 1.86 & 1.76 \\
64QAM & $2 / 3$ & 22.7 & 4.0 & 2.77 & 2.48 & 2.35 \\
64QAM & $3 / 4$ & 24.4 & 4.5 & 3.11 & 2.79 & 2.63 \\
\hline \hline
\end{tabular}

TABLE V

DESIGN OPTIONS, $r=1 \mathrm{KM}$.

\begin{tabular}{l|l|l|l|c}
\hline \hline Situation & $N$ & $\begin{array}{l}R_{\text {net }} \\
(b / s / H z)\end{array}$ & $\begin{array}{l}A A S E \\
\left(b / s / H z / \mathrm{km}^{2}\right)\end{array}$ & $\begin{array}{c}\text { Antenna } \\
\text { Config. }\end{array}$ \\
\hline $\begin{array}{l}\text { NLOS path } \\
\text { no SR } \\
n_{\text {hops }}=1\end{array}$ & 3 & 0.880 & 0.073 & 5 \\
\hline $\begin{array}{l}\text { NLOS paths } \\
\text { no SR } \\
n_{\text {hops }}=2\end{array}$ & 3 & 0.749 & 0.062 & 5 \\
\hline $\begin{array}{l}\text { NLOS paths } \\
\text { no SR } \\
n_{\text {hops }}=3\end{array}$ & 3 & 0.797 & 0.066 & 5 \\
\hline $\begin{array}{l}\text { NLOS paths } \\
\text { SR } \\
n_{\text {hops }}=3\end{array}$ & 3 & 1.533 & 0.13 & 5 \\
\hline $\begin{array}{l}\text { LOS paths } \\
\text { no SR } \\
n_{\text {hops }}=3\end{array}$ & 1 & 1.036 & 0.26 & 5 \\
\hline $\begin{array}{l}\text { LOS paths } \\
\text { SR } \\
n_{\text {hops }}=3\end{array}$ & 1 & 1.992 & 0.50 & 5 \\
\hline \hline
\end{tabular}

TABLE VI

DESIGN OPTIONS WITH OMNIDIRECTIONAL SS, $r=1 \mathrm{KM}$.

\begin{tabular}{l|l|l|l|c}
\hline \hline Situation & $N$ & $\begin{array}{l}R_{\text {net }} \\
(b / s / H z)\end{array}$ & $\begin{array}{l}A A S E \\
\left(b / s / H z / \mathrm{km}^{2}\right)\end{array}$ & $\begin{array}{c}\text { Antenna } \\
\text { Config. }\end{array}$ \\
\hline $\begin{array}{l}\text { NLOS path } \\
\text { no SR } \\
n_{\text {hops }}=1\end{array}$ & - & 0.000 & 0.00 & 4 \\
\hline $\begin{array}{l}\text { NLOS paths } \\
\text { no SR } \\
n_{\text {hops }}=2\end{array}$ & 3 & 0.489 & 0.041 & 4 \\
\hline $\begin{array}{l}\text { NLOS paths } \\
\text { no SR } \\
n_{\text {hops }}=4\end{array}$ & 3 & 0.784 & 0.065 & 4 \\
\hline $\begin{array}{l}\text { NLOS paths } \\
\text { SR } \\
n_{\text {hops }}=4\end{array}$ & 3 & 1.537 & 0.128 & 4 \\
\hline $\begin{array}{l}\text { LOS paths } \\
\text { no SR } \\
n_{\text {hops }}=3\end{array}$ & 2 & 1.036 & 0.130 & 4 \\
\hline $\begin{array}{l}\text { LOS paths } \\
\text { SR } \\
n_{\text {hops }}=3\end{array}$ & 2 & 1.992 & 0.249 & 4 \\
\hline \hline
\end{tabular}

Table VII and Fig. 3 show the performance, $R_{\text {net }}$ and $A A S E$, for various macrocell sizes and relay hops. These designs have been chosen for comparison since they maximize the $A A S E$ for each case. The breakpoint is assumed to be $250 \mathrm{~m}$. Note again that the addition of one relay (creating two hops) decreases the network throughput since additional resources (time slots) are required and spatial reuse is not possible. There is an improvement for all cell sizes with the introduction of a second relay (three hops) since spatial reuse and a reduction in pathloss occur. Note from the Fig. 3(a) that there is apparently little improvement in the network throughput of three hops over one hop, but Fig. 3(b) shows a great increase in $A A S E$ due to the decrease in cluster size.

\section{CONCLUSIONS}

Due to the high cost of using licensed frequencies, many wireless service providers are interested in unlicensed bands. The use of the unlicensed band creates additional difficulties in designing multi-hop relaying in 802.16/HiperMAN systems because there are strict limits on transmit power, high levels of interference, and higher pathloss in the $5 \mathrm{GHz}$ range. We have presented calculations of network spectral efficiency, comparing area averaged spectral efficiency (AASE), to determine suitable designs. We show that the use of AASE is a more appropriate measure of system design efficiency than network throughput. AASE takes into consideration the macrocell cluster size in determining the efficient use of spectrum, whereas network throughput does not. Several conclusions can be drawn from the results presented here. The addition of relays into an existing cellular system may in some cases provide little or no advantage since additional resources (time or frequency slots) are required to perform relaying. Advantage is gained by introducing spatial reuse within a macrocell and by significantly reducing the path loss. The most significant gain is achieved by adding enough relays to create LOS relay hop paths where NLOS would have occurred with no relays. Depending on the initial macrocell size, this may require three or more relay hops. The number of hops achieving the best AASE depends greatly on the specific propagation environment, and the dimensions of macrocell. Although the relay hops are LOS, interference paths remain NLOS, so a much smaller cluster size, $N$, can be used, which increases AASE. 
TABLE VII

$R_{n e t}(b / s / H z)$ AND $A A S E\left(b / s / H z / k m^{2}\right)$ FOR VARIOUS SCENARIOS WITH SPATIAL REUSE (WHEN POSSIBLE). BLACK FONT INDICATES AT LEAST ONE HOP IS NLOS, GREEN FONT INDICATES ALL HOPS ARE LOS.

\begin{tabular}{c|ccc|ccc|ccc|ccc|}
\hline \hline Radius $\rightarrow$ & \multicolumn{3}{|c|}{$3.0 \mathrm{~km}$} & \multicolumn{4}{c|}{$2.0 \mathrm{~km}$} & \multicolumn{3}{c|}{$1.0 \mathrm{~km}$} & \multicolumn{3}{c}{$0.5 \mathrm{~km}$} \\
$n_{\text {hops }}$ & $R_{\text {net }}$ & $N$ & AASE & $R_{\text {net }}$ & $N$ & AASE & $R_{\text {net }}$ & $N$ & $A A S E$ & $R_{\text {net }}$ & $N$ & $A A S E$ \\
\hline 1 & 0.000 & - & 0.000 & 0.000 & - & 0.000 & 0.880 & 2 & 0.074 & 1.760 & 4 & 0.440 \\
2 & 0.000 & - & 0.000 & 0.000 & - & 0.000 & 0.520 & 2 & 0.065 & 1.198 & 3 & 0.399 \\
3 & 0.000 & - & 0.000 & 0.606 & 2 & 0.019 & 1.533 & 3 & 0.128 & 1.992 & 1 & 1.992 \\
4 & 0.236 & 2 & 0.003 & 0.670 & 2 & 0.021 & 1.186 & 2 & 0.148 & 1.591 & 1 & 1.591 \\
\hline
\end{tabular}

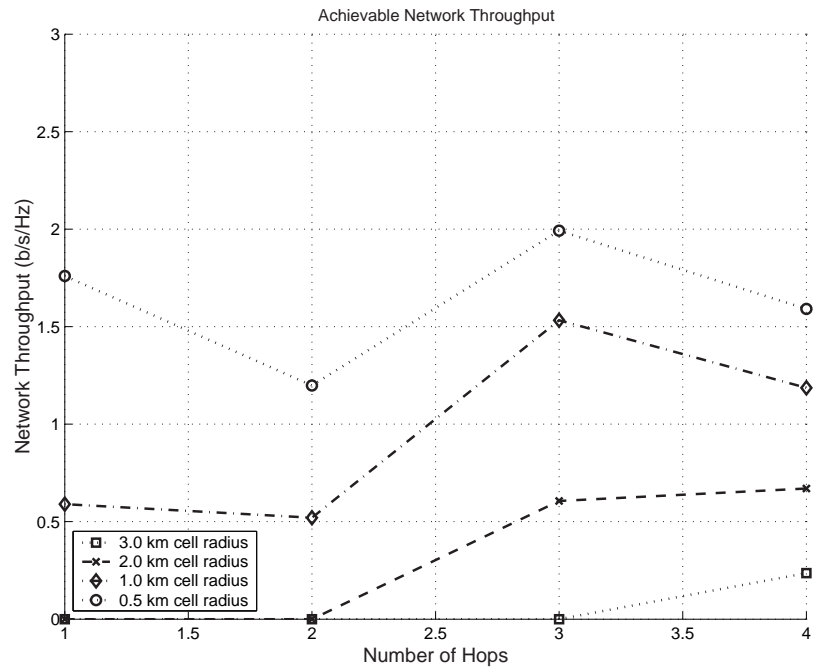

(a) $R_{\text {net }}$ per macrocell.

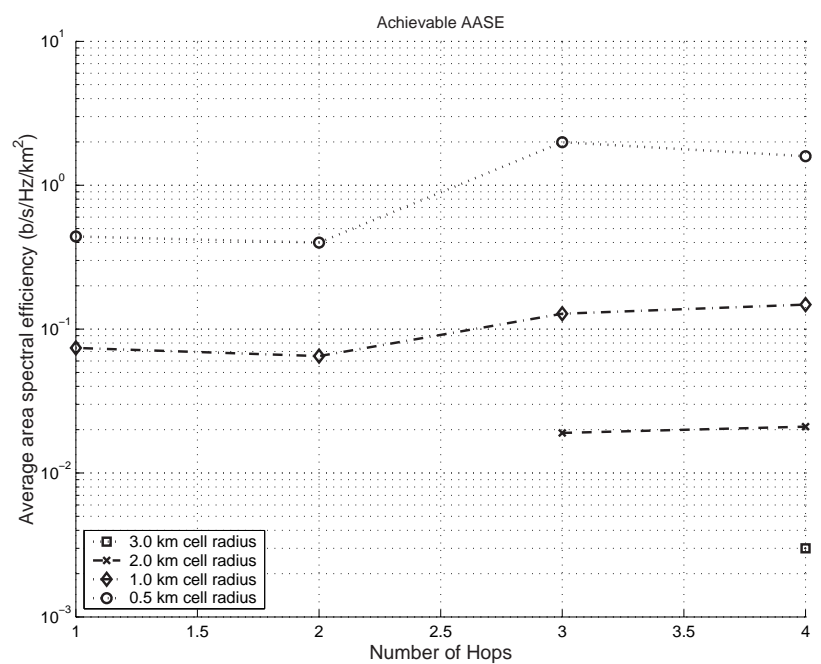

(b) System $A A S E$.

Fig. 3. Network performance for Manhattan layout, $250 \mathrm{~m}$ breakpoint.

\section{ACKNOWLEDGEMENTS}

The authors gratefully acknowledge funding for this work provided by the Natural Sciences and Engineering Research Council (NSERC) of Canada, TRLabs, Rohit Sharma Professorship, Alberta Informatics Circle of Research Excellence (iCORE), TELUS Mobility, and the Canadian Council of Professional Engineers (CCPE).

\section{REFERENCES}

[1] D. C. Schultz, R. Pabst, and T. Irnich, "Multi-hop based radio network deployment for efficient broadband radio coverage," in Proc. WPMC'03, Yokosuka, Japan, October 2003, pp. 377-381.

[2] N. Esseling, B. Walke, and R. Pabst, "Fixed relays for next generation wireless systems," in Emerging Location Aware Broadband Wireless Ad Hoc Networks, R. Ganesh, S. Kota, K. Pahlavan, and R. Agustí, Eds. New York, USA: Springer Science+Business Media, Inc., 2005, ch. 5, pp. 71-91.

[3] H. Yanikomeroglu, "Fixed and mobile relaying technologies for cellular networks," in Proc. Second Workshop on Applications and Services in Wireless Networks (ASWN 2002), July 2002, pp. 75-81.

[4] L. Kleinrock and J. Silvester, "Spatial reuse in multihop packet radio networks," in Proc. the IEEE, vol. 75, no. 1, January 1987, pp. 156-167.

[5] J. Grönkvist, J. Nilsson, and D. Yuan, "Throughput of optimal spatial reuse TDMA for wireless and ad-hoc networks," in Proc. VTC2004Spring, Milan, Italy, May 2004, pp. 2156-2160.

[6] IEEE Standard for Local and Metropolitan Area Networks - Part 16: Air Interface for Fixed Broadband Wireless Access Systems, IEEE Std. 802.16-2004.
[7] M. Nohara, K. Saito, K. Sugiyama, H. Shinonaga, J. Cho, J. Son, H. Lee, N. Natarajan, D. T. Chen, and M. Asa, "Mobile multi-hop relay networking in IEEE 802.16," IEEE, Tech. Rep. IEEE-C802.16-05/013, July 2005

[8] K. Saito, M. Nohara, and K. Sugiyama, "Study of IEEE 802.16 mobile multi-hop relay," IEEE, Tech. Rep. IEEE-C802.16-05/001, September 2005.

[9] "Broadband radio access networks (BRAN); HiperMAN; physical (PHY) layer," ETSI, Tech. Rep. TS-102-177 V1.3.1, February 2006.

[10] "Broadband radio access networks (BRAN); HiperMAN; data link control (DLC) layer," ETSI, Tech. Rep. TS-102-178 V1.3.1, February 2006.

[11] "Specifications for $2.3 \mathrm{GHz}$ band portable internet service - physical layer," Telecommunications Technology Association (TTA), Tech. Rep. TTAS.KO-06.0064R1, 2004.

[12] "Universal mobile telecommunications system (UMTS); spatial channel model for multiple input multiple output (MIMO) simulations," ETSI, Tech. Rep. 3GPP TR 25.996 Version 6.1.0 Release 6, September 2003.

[13] D. S. Baum et al., "An interim channel model for beyond-3G systems," in Proc. VTC'05-Spring, Stockholm, Sweden, May 2005, pp. 3132 3136.

[14] K. Hole and G. E. Oien, "Spectral efficiency of adaptive coded modulation in urban microcellular networks," IEEE Trans. Veh. Technol., vol. 50, no. 1, pp. 205-222, January 2001.

[15] M.-S. Alouini and A. Goldsmith, "Area spectral efficiency of cellular mobile radio systems," IEEE Trans. Veh. Technol., vol. 48, no. 4, pp. 1047-1066, July 1999.

[16] V. Erceg et al., "Channel models for fixed wireless applications," IEEE, Tech. Rep. IEEE-C802.16.3c-01/29r4, July 2001.

[17] J. Khun-Jush, P. Schramm, U. Wachsmann, and F. Wenger, "Structure and performance of the HiperMAN/2 physical layer," in Proc. VTC1999Fall, Amsterdam, The Netherlands, September 1999, pp. 19-22.

[18] C. Hoymann and B. Walke, "WiMAX Wireless Metropolitan Area Networks," in European Wireless 2005 (Tutorial), April 2005 